\section{Abstractions}

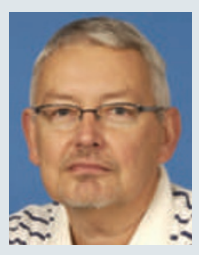

\section{FIRST AUTHOR}

Climate is never static, but it does veer between relatively steady states and periods of drastic fluctuation. During the past 3 million years, climate has become increasingly variable. About 1 million years ago, the magnitude of glacial-interglacial oscillations increased significantly, with two of the most severe glaciations of the 3-millionyear period occurring in the past 150,000 years. These nonlinear events are generally attributed to subtle cyclical changes in Earth's orbit or variations in atmospheric carbon dioxide concentrations. Thomas Crowley, a geoscientist at the University of Edinburgh, UK, and William Hyde, a physicist at the University of Toronto in Canada, suggest that this picture is incomplete. On page 226 , they show how models of past climate transitions bolster their suggestion that the more variable recent climate may be an indication of an imminent - in geological terms - shift to a new steady state comprising stable midlatitude ice sheets. But, Crowley tells Nature, anthropogenic increases in $\mathrm{CO}_{2}$ make any such transitions unlikely.

How did your climate modelling experience lead you to this hypothesis?

Twenty years ago, I noticed enhanced variability around climate transitions in very simple climate models. This stuck in my mind, and one day a few years ago I started tinkering with data to look for similar trends in ice-sheet fluctuations during the past half a million years. Since then, we've tested more realistic models, including ones for icesheet dynamics, to see whether they could simulate that enhanced variability. They did.

\section{What causes the variability?}

In some ways, it's analogous to variability in the financial markets. Conditions tend to be more volatile during times of rapid change. As the climate system approaches a more stable state, such as an ice-sheet expansion, the system varies even more. With respect to the current climate, we're suggesting that the system may be oscillating because it is approaching a stable state. If Earth's climate had not been interfered with by humans, our model suggests the transition could have happened within the next 100,000 years.

\section{So humans might inadvertently have} prevented the next ice age?

It's possible. At the moment, global warming is occurring at a much faster rate than is any transition likely to cause a climate switch in the opposite direction. The amount of $\mathrm{CO}_{2}$ in the atmosphere is already more than enough to prevent Earth from reaching a state with considerable ice cover. Some might argue that we should continue to pollute to prevent an ice age, but this is not a valid argument.

\section{MAKING THE PAPER}

\section{Michale Fee}

\section{An innovative cooling device chills a brain circuit and slows birdsong.}

One way to better understand how brain circuits control behaviour is to take recordings from as many individual brain cells as possible. Making single-neuron recordings in an awake animal as a behaviour unfolds is tricky, and the hard work doesn't necessarily pay off: in animals with complex brain circuits that contain mutual connections, the technique doesn't always give clear results. And for Michale Fee, a neuroscientist at the Massachusetts Institute of Technology in Cambridge, the process is further complicated by his temperamental subject, a 15-gram songbird that won't sing if handled too much.

To get around these obstacles, Fee and his postdoc, Michael Long, devised a simple but innovative contraption to study how a zebra finch's brain controls the timing of its singing.

Male zebra finches sing one song throughout life - a song comprising a repeating motif that sounds a bit like a squeaky wheel and lasts for up to about one second. A song's length depends on several factors, including how many motifs are repeated, the timing between motifs, the duration of the syllables that make up the motif and the timing between each syllable. Data from a study done in 2002 suggested that a brain area called the premotor nucleus HVC (HVC) acts as the clock for at least one aspect of song timing.

But simply observing HVC neurons firing during a song would not be conclusive, says Fee. "You can't distinguish which neurons are generating the timing and which neurons are following the timing just by watching them because these circuits have mutual connections between neurons," he says.

Long had begun investigating how HVC cells generate their regular, clock-like bursts of activity. Brain areas can be reversibly inactivated by cooling them to about $10^{\circ} \mathrm{C}$, and Long and Fee discussed using temperature to temporarily

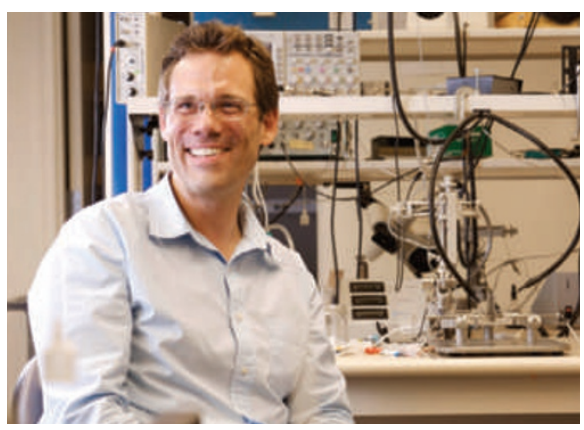

block the activity of the HVC. It dawned on Fee that less drastic action - cooling the HVC by just a few degrees - could be a good test for the control of song timing.

"Temperature has a very strong effect on the speed at which neurons operate," explains Fee. "We realized we could use temperature to very subtly control the speed of the circuit and see whether that affected the timing of singing." The experiment required a device that could precisely cool the HVC but that would still allow finches to move and sing freely. Fortunately, the HVC is near the brain surface and Fee is handy at engineering tiny devices.

He disassembled a thermoelectric device one that converts an electric current into a temperature difference - and used its components to build two tiny gold-plated probes to place on both the right and left hemisphere HVCs in a finch's brain. He and Long then tested the instrument. "We ran the current through the cooling device, put a female in front of a male, he started singing and it came out more slowly," recalls Fee. "It was amazing. The effect was very clear."

All components of the song's timing seem to be controlled by the HVC, and stretch out by about $3 \%$ for every $1{ }^{\circ} \mathrm{C}$ of cooling. Long and Fee propose that the HVC clock operates similarly to a set of dominoes, with each neuron kicking off the next one to fire, and then resetting itself for the next song motif (see page 189).

Fee says his invention should be a powerful way to study questions about how brain circuits control timing. But, he adds, his gizmo-building is exclusively reserved for the lab. "I'm surprisingly resistant to home repairs.”

\title{
FROM THE BLOGOSPHERE
}

In its November editorial,

Nature Reviews Microbiology $(6,794 ; 2008)$ reports that the archive of the International Journal of Systematic and Evolutionary Microbiology (IJSEM) has been made available free online: a boon for scientists, historians and the public. As outlined on Nautilus, the value of systematics is often underappreciated for bacteria and viruses

\section{(http://blogs.nature.com/} nautilus/2008/10/historical_ microbiology_archiv.html).

These are the most populous organisms on Earth, although more than $99 \%$ of bacteria have yet to be cultivated. Naming and characterizing different species of bacteria, and the investigation of the relationships between them, is an important adjunct to genomics-based approaches, which are producing everincreasing estimates of the numbers and types of these organisms.

Jean Euzeby, the IJSEM

list editor, maintains a web resource that details all those species that have been ratified by an international committee. Links to this and other useful resources and websites are provided in the Nautilus post. 